\title{
Neuropsychological rehabilitation, neuroimaging and neuroplasticity: A clinical commentary
}

\author{
George P. Prigatano $^{\mathrm{a}, *}$, Lucia W. Braga ${ }^{\mathrm{b}}$, Spring Flores Johnson ${ }^{\mathrm{a}}$ and Lígia M.N. Souza ${ }^{\mathrm{b}}$ \\ ${ }^{a}$ Department of Clinical Neuropsychology, Barrow Neurological Institute, St. Joseph's Hospital and Medical \\ Center, Phoenix, AZ, USA \\ ${ }^{\mathrm{b}}$ SARAH Network of Rehabilitation Hospitals, Brasilia, Brazil
}

\begin{abstract}
Initial brain imaging studies on recovery of motor functioning after stroke suggested their potential prognostic value in neurorehabilitation. However, the value of brain imaging in documenting brain changes associated with cognitive and behavioral treatment effects seem less likely. Also, neuroimaging studies at that time seem to have little, if any, value for treatment planning. Advances in neuroimaging technology are beginning to challenge these initial impressions. In this clinical commentary, we propose that advances in the field of neuroimaging have relevance for the future development of neuropsychological rehabilitation. Neuropsychological rehabilitation is entering a new era that involves collaboration with neuroimaging and associated studies on neuroplasticity. We recognize that this may seem "aspirational" rather than practical in most rehabilitation settings. However, we provide examples of how this can be achieved as illustrated by collaborative efforts of clinicians and scientists in the SARAH Network of Rehabilitation Hospitals in Brazil. We also review selective papers on neuroplasticity, spontaneous recovery and diaschisis that have relevance for research which will expand and further develop the field of neuropsychological rehabilitation.
\end{abstract}

Keywords: Neuropsychological rehabilitation, neuroplasticity, diaschisis, neuroimaging

\section{Introduction}

Efforts to help individuals with a history of brain injury improve their neuropsychological functions have a rather long history, as briefly summarized by Newcombe (2002). While Kurt Goldstein was rightly describe as the "great precursor" to neuropsychological rehabilitation, many individuals have significantly contributed to addressing the complex topic of how to help patients improve or partially remediate impaired higher integrative brain functions. They include such

\footnotetext{
*Address for correspondence: George P. Prigatano Ph.D., Barrow Neurological Institute, Department of Clinical Neuropsychology, 222 West Thomas Road, Suite 315, Phoenix, AS 85013, USA. Tel.: +1 602406 3671; Fax: +1 602406 6115; E-mail: george.prigatano@dignityhealth.org.
}

historical figures as Oliver Zangwill (1947) and A.R. Luria (1948/1963). Newcombe (2002) also makes the important observation that neuropsychological rehabilitation has developed as a result of its collaboration with other disciplines of study, including experimental psychology, cognitive neuropsychology, and learning theory. One might also add that holistic approaches to neuropsychological rehabilitation have made substantial contributions (Ben-Yishay \& Diller, 2011).

The rehabilitation of persons with neuropsychological impairments has remained, however, a demanding task for at least two reasons. First, understanding the functional neuroconnectivity patterns of activation/deactivation that underlie these difficult to remediate impairments has remained a challenging 
task despite some impressive advances in the neurosciences and neuroimaging of the brain (Siegel et al., 2016; Warren et al., 2014). Second, having a methodology with associated technology to systematically remediate such complicated higher brain functions as attention, memory, working memory, and various "executive functions" has been lacking. Several years ago, Luria and his colleagues (Luria et al., 1969), insightfully suggested that the process of restoring higher integrative brain functions could be achieved by systematic cognitive-behavioral efforts to "deinhibit" regions of neural activity that were rendered temporally dysfunctional via the process of diaschisis. This proved to be, however, a more demanding task than was anticipated.

Due to this reality, teaching patients to compensate for disabilities related to neuropsychological impairments became the most practical approach to helping them. This approach was again seen in the early work of Goldstein (1942) and Zangwill (1947). Nevertheless, the process of helping the patient systematically use compensatory strategies was not without difficulty. Patients wanted a return to normal functioning (Prigatano, 1995). They were understandably frustrated and discouraged when it became clear this was not going to happen. Problems of depression, angry outbursts, and catastrophic reactions made it clear to Goldstein (1954) that some form of psychotherapy would be a necessary component of neuropsychological rehabilitation for some patients.

While the use of various forms of psychotherapy is now a part of many programs aimed at neuropsychological rehabilitation, the introduction of formal psychotherapy activities into the rehabilitation of persons with a brain disorder was approached with caution. There were many negative experiences in which the behavioral problems of persons with underlying brain dysfunction were erroneously interpreted as due to underlying psychodynamic conflicts versus being a direct or indirect effect of the brain disorder (Prigatano, 2008). Instead of utilizing traditional forms of psychotherapy, the development of systematic and well thought out social group support activities was seen as a necessary and preferred method of helping patients emotionally adjust to the permanent effects of brain disorder. Thus the "milieu approach" advocated by Ben Yen-Yishay and Diller (2011) became the prototype for effective holisticoriented neuropsychological rehabilitation.

Neuropsychological rehabilitation after a brain injury is now entering a new era. Besides studying possible improvements on neuropsychological tests, assessing the patients' emotional and social adaption, and determining if they return to a productive life style, the "evidence" that meaningful changes in brain function do occur as a result of rehabilitation experiences requires direct observations of how the person's brain is responding to treatments (Nordvik et al., 2014; Katz \& Dwyer, 2021). Directly linking behavioral and cognitive changes to brain changes is one of the important next steps in the development of neuropsychological rehabilitation. This is a difficult step to achieve given the financial costs and the need of collaborative efforts of many skilled individuals working in the areas of neuroplasticity, neuroimaging and neuropsychological rehabilitation. However, achieving this goal of directly linking cognitive and behavioral changes to changes in brain function helps improve our scientific understanding of the mechanisms that underlie complete versus partial recovery of different higher brain functions. It also provides potential evidence that more than compensatory training may occur as a result of different rehabilitation activities.

In this clinical commentary, we summarize the clinical and research findings on neuroimaging evidence of neuroplasticity for improved neuropsychological functions as reported by the SARAH Network of Rehabilitation Hospitals in Brazil, as an example of what can be done. The findings reported are encouraging and suggest that combining well-orchestrated neuropsychological rehabilitation activities with neuroimaging studies can reveal evidence of meaningful changes attributed to neuroplasticity. In addition, we comment on a few recent observations reported in the literature on the phenomena of neuroplasticity, spontaneous recovery and diaschisis that are relevant for advancing the field of neuropsychological rehabilitation. We begin, however, with a brief review of studies on neuroimaging correlates of motor recovery after stroke that potentially provides a limited perspective on the role of neuroimaging in neurorehabilitation. We then review a definition of neuroplasticity and how it may be related to spontaneous recovery after a brain insult.

\section{Initial studies of neuroimaging and motor recovery}

Corbetta (2008) summarized the various scientific hurdles that need to be overcome when related 
neuroimaging changes to recovery of various neurological functions, including motor functioning. In summarizing early research in this area, he notes that neuroimaging studies allow for "visualization of neural mechanisms of recovery at the systems level" (pg.163). Research at that time revealed two important observations. First, different mechanisms of recovery can be observed and frequently involve the recruitment of bilateral brain regions not directly connected to the motor system. This implied that some form of behavioral (as well as neural) compensation may underlie what appears to be "recovery" of impaired motor functions. The second, and perhaps the most important observation, was that a return to normal brain activation patterns, when performing motor tasks, was always associated with the best recovery. This suggested it was not neuroplasticity per se that was occurring, but rather spontaneous recovery of a once impaired motor network of the brain. These findings led many to consider neuroimaging as a potential useful tool for prognosis, but not in documenting neuroplasticity phenomena much less aiding in treatment planning (Stinear \& Ward, 2013).

\section{A definition of neuroplasticity and the phenomenon of spontaneous recovery}

A National Institutes of Health conference (Cramer et al., 2011) proposed the following working definition of neuroplasticity: "Neuroplasticity can be broadly defined as the ability of the nervous system to respond to intrinsic and extrinsic stimuli by reorganizing its structure, function and connections; can be described at many levels, from molecular to cellular to systems to behavior; and can occur during development, in response to the environment, in support of learning, in response to disease or in relation to therapy" (p. 1592). The core feature of this well thought out description is that the central nervous system can be directly changed in its "structure, function and connections" by naturally occurring biological changes and external stimulation or learning experiences. It is important to note that neuroplasticity does not refer to compensatory activities of the brain (and associated neuropsychological functions), but to some actual re-structuring or re-organization that influences brain activity at multiple levels.

Including "intrinsic" stimuli as a modifying agent suggests that some aspects of spontaneous recov- ery may also reflect a neuroplasticity phenomenon. Cassidy and Cramer (2017) note the recovery of neurological (including neuropsychological) functions often entails "the restitution of function in injured but surviving neural matter" (pg.33). How this restitution occurs has been studied at the cellular and molecular levels and appears to include such phenomena as growth of synapses and dendrites, axonal remodeling and increased expression of growth-related genes and proteins to spontaneously enhance brain alterations (see Cassidy and Cramer, 2017 for a more detail discussion)

The classical "external" stimuli which promote neuroplasticity are thought to include physical and cognitive rehabilitation exercises that provide learning experiences that aim at modifying brain function and structure in response to damaged (not undamaged) areas of the brain. With the advent of pharmacological interventions and transcranial magnetic stimulation (TMS), the potential list of "external" stimuli is expanding in the field of neurorehabilitation and ultimately will influence neuropsychological rehabilitation (Cassidy and Cramer, 2017; Katz \& Dwyer, 2021).

\section{Neuroplasticity and neuroimaging}

Starting in the 1980s, new techniques for brain imaging and neurophysiology permitted a deeper exploration of how daily life affects the human brain. The development of advanced technologies such as positron emission tomography (PET), functional magnetic resonance imaging (fMRI), diffusion tensor image (DTI), event-related potential (ERP), and magnetoencephalography (MEG) have contributed significantly to a growing scientific understanding of how changes in brain structure and functional connectivity are relevant to neuropsychological rehabilitation (Rios-Lago et al., 2004). These techniques have been shown to help measure and monitor the impact of rehabilitation interventions on neuroplasticity (Stinear \& Ward, 2013; Nordvik et al., 2014). With functional neuroimaging, visualizing brain activity associated with various perceptive, $\operatorname{cog}$ nitive, emotional, and behavioral processes in both normal subjects and those with brain injury is now possible. Neuroimaging findings can be recorded before, during, and after rehabilitation. As such, they can help gauge the efficacy of rehabilitative exercises and help design more personalized treatment 
programs while monitoring longitudinal structural changes (Munos Cespedes, 2005; Strangman et al., 2005; Hamaide, 2016; Heiss, 2017; Caeyenberghs et al., 2018).

Studies on human neuroplasticity initially focused on infancy and analyzed the process of brain maturation, myelination, and changes in the brain due to natural biological development. Subsequently, brain imaging techniques began to illuminate neuroplasticity phenomena in different periods of life and framed this in specific types of learning. More recent investigations have revealed that the adult brain can adaptively alter its structure (anatomic plasticity/white matter) and functional organization (functional plasticity/gray matter) in response to daily experiences (Zatorre et al., 2013).

Despite being less flexible than during the initial phases of development, cortical plasticity is evident throughout adulthood, in terms of both structure and function (Braga et al., 2017; De Schotten et al., 2014; Dehaene et al., 2011). Neuroplasticity permits the adult brain to acquire and retain memory in later phases of life and to "remold" itself after cognitive and physical training (Hamaide, 2016; Galetto $\&$ Sacco, 2017).

\section{Neuroimaging, neuroplasticity and literacy training in normal adults at the SARAH Network of Rehabilitation Hospitals}

Turkeltaub et al. (2003) used fMRI to study subjects between 6 and 22 years of age and showed the brain's capacity for reorganization after literacy training. The brain evolves during literacy acquisition in adulthood (Braga et al., 2017; Dehaene, Cohen, Morais, \& Kolinsky, 2015; Abutalebi et al., 2007). Written language processing is, in most individuals, located in the left hemisphere, but this lateralization only occurs after training and proficiency in a given skill. In childhood, before learning to read and write, the child uses the area known as the visual word form area (VWFA), located in the occipito-temporal transition of the left hemisphere to recognize faces. After learning to read, face recognition migrates to the corresponding area in the right hemisphere, and the VWFA, in the left hemisphere, is recycled and used in the recognition of letters. This migration is seen not only in children but also in individuals who learned to read and write as adults (Dehaene et al., 2010).
Illiteracy has a significant impact on cognitive function and its regions in the brain (Ardila et al., 2010; Castro-Caldas, Reis e Guerreiro, 1997). Consequently, a comparative study of literate and illiterate subjects that follows the literacy acquisition process in illiterate adults was a unique opportunity to explore the impact of the interaction between neurobiological factors and learning a specific proficiency. The SARAH Network of Rehabilitation Hospitals, which has nine hospitals in Brazil, has established collaborative efforts with other international research institutions in order to conduct comparative studies of literate and illiterate subjects. One major goal was to explore the impact that literacy acquisition has on the brain of healthy illiterate adults, who for sociocultural reasons, were unable to learn to read and write in childhood (Dehane et al., 2010). The results obtained corroborated the finding that the adult brain can adaptively change its structure (anatomical neuroplasticity) and functional organization (functional neuroplasticity) in response to experiences that promote the enrichment of the environment in which they live. Functional MRI was used to compare brain activity in literate adults $(n=31)$, illiterate adults $(n=10)$, and unschooled subjects who learned to read and write in adulthood (previously illiterate adults $n=22$ ). Brain activity in response to a series of stimuli (i.e., spoken and written phrases, spoken words and pseudo words, and picture of faces, houses, objects, and checkerboards) were obtained. It was noted that as literacy levels advanced, activation increased in the left occipito-temporal lobe transition, evoked by writing, in the VWFA and broadly increased visual responses in the fusiform and occipital cortex, extending all the way to the V1 area. Literacy also increased phonological speech activation in the planum temporale and permitted a top to bottom activation of orthography from spoken entries. Most of these changes occurred even when literacy was acquired in adulthood, underscoring the fact that schooling in childhood as well as adulthood can profoundly alter cortical organization. The study added to previous research that examines children before and after literacy acquisition, but with the added advantage of excluding the issue of childhood brain maturation. In the case of illiterate adults, brain plasticity was enough to allow literacy acquisition to provoke changes in functional (Dehaene et al., 2010) and anatomical (De Schotten et al., 2012) connections. Literacy, whether acquired in childhood or adulthood, boosts the organization of visual cortices, particularly by inducing an enhanced response to the known script at the VWFA 


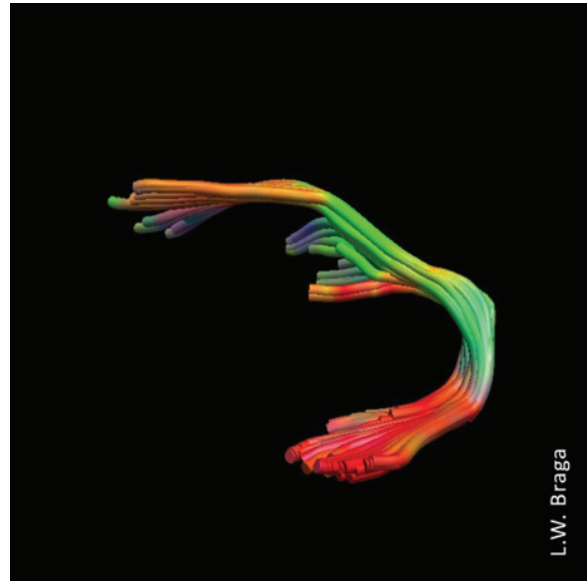

Fig $1 \mathrm{a}-$ left arcuate fasciculus before literacy

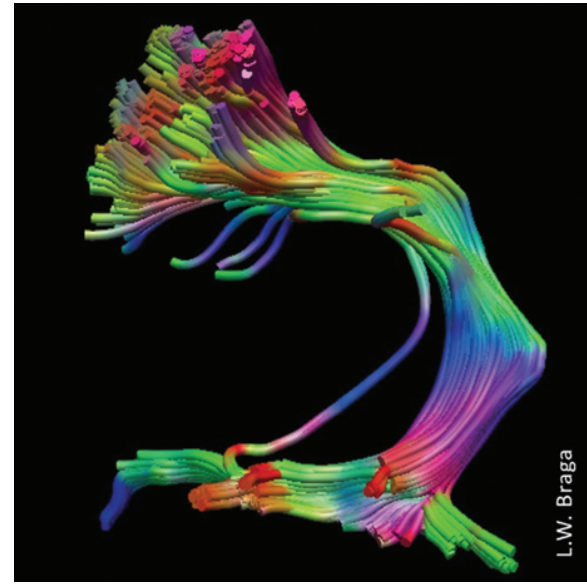

Fig $1 \mathrm{~b}$ - left arcuate fasciculus after literacy

Fig. 1. DTI images of the participant's left arcuate fasciculus (a) before and b) after intervention. There was a significant increase in number of fibers, volume, and length of the left arcuate fasciculus after literacy acquisition.

site in left occipito-temporal cortex and by augmenting early visual responses in the occipital cortex, in a partially retino topic manner. It permits, practically, the entire left-hemispheric spoken language network to be activated by written sentences and refines spoken language processing by enhancing a phonological region, the planum temporale, and by making an orthographic code available in a top-down manner.

Braga et al. (2017) demonstrated that adult plasticity is sufficient for later acquisition of literacy, in the absence of any schooling or literacy learning in childhood. The authors evaluated the neurofunctional changes associated with late literacy acquisition in an illiterate adult who underwent 20 fMRI exams over the course of two years, while progressing from complete illiteracy to a level of alphabetic decoding. Initially, the participant did not activate neural circuits for reading when he was exposed to words; gradually, however, he began to present activation in the left occipito-temporal cortex, at the visual word form area. This increase was accompanied by a decrease in face responses in the left VWFA and increase in the right hemisphere. Reading-related responses also emerged in language-related areas of the inferior frontal gyrus and temporal lobe. Additional activations in the superior parietal lobe, superior frontal gyrus and posterior medial frontal cortex suggested that reading remained dependent on effortful executive attention and working memory processes. Nevertheless, the results indicate that adult plasticity can be sufficient to induce rapid changes in brain responses to written words and faces in an unschooled and illiterate adult. They also demonstrate that literacy acquisition requires greater participation of the right hemisphere during the initial stages as well as activation of the frontal areas, which may be associated with learning. This subject underwent a DTI study of the tracts that interconnect the brain, and a comparison of the fractional anisotropy (FA) from before and then after literacy acquisition was done. There was a significant increase in connectivity of the arcuate fasciculus (AF), which connects the Broca and Wernicke areas. Literacy promoted important changes in white matter; the clinical intervention of learning to read yielded neuroplasticity outcomes in the AF (Fig. 1).

These results, which highlight increased brain connectivity by enriching a subject's environment with interventions such as literacy acquisition, have encouraged clinicians and researchers to study the impact of neuropsychological rehabilitation on neuroplasticity.

\section{Neuroimaging, neuroplasticity and neuropsychological rehabilitation after stroke: A case report from the SARAH Rehabilitation Network in Brazil}

The use of functional neuroimaging and tractography has been increasingly applied to the evaluation of rehabilitation outcomes in patients with stroke (Carey \& Seitz, 2007, Johansen-Berg, 2007; Gale \& Pearson, 2012; Stinear \& Ward, 2013; Heiss, 2017) and TBI (Strangaman et al., 2005 Galetto \& Sacco, 2017, 

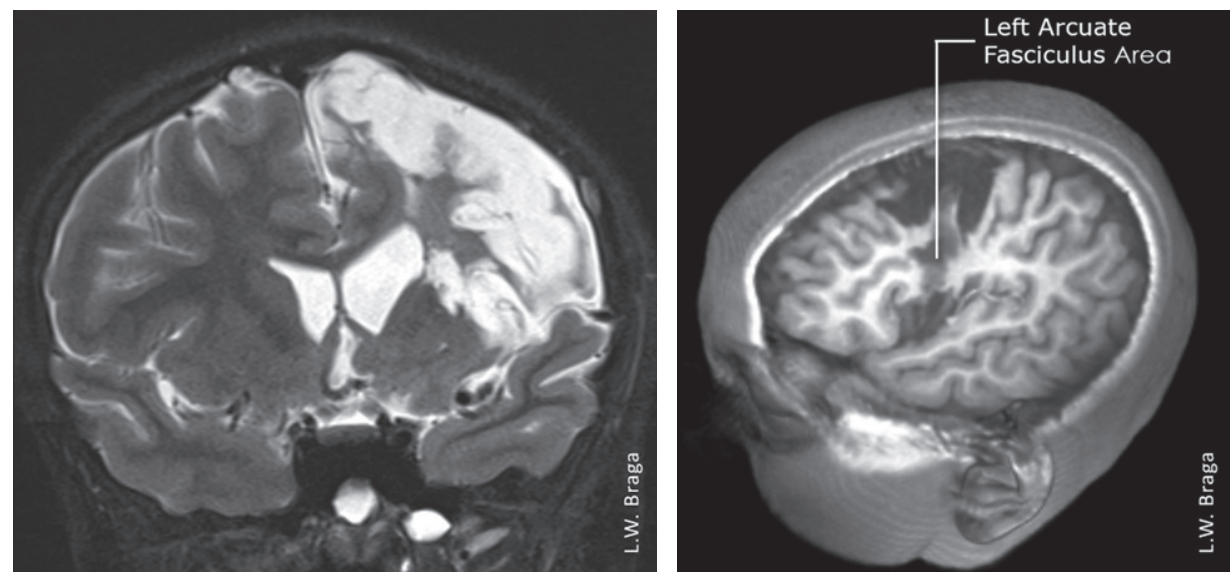

Fig. 2. MRI images of TF ten months after injury, showing extensive lesion in the left cerebral hemisphere affecting brain areas responsible for language. There was an important lesion in the left arcuate fasciculus.

Caeyenberghs et al., 2018). A frequent question is whether neuroplasticity occurs only during the acute phase of a brain injury, or whether, even in the chronic stage, the brain can undergo functional and structural changes as a result of neurorehabilitation. To further explore this issue, neuropsychological rehabilitation aimed at restoring language functions was attempted with an adolescent who was in the chronic stage of her post-stroke recovery.

TF was an 11-year-old bilingual (Farsi/Portuguese) right-handed girl who suffered from an acute stroke of the left middle cerebral artery (MCA) due to embolism from an intracardiac tumor in October 2016. An echocardiogram showed a tumor (myxoma) in the left atrial cavity, which was surgically removed 20 days later. She initially presented with total paralysis of the right side, anomic aphasia, and alexia. Restorative language training was attempted 10 months post-onset of TF's stroke at the Brasilia unit of the SARAH Network of Neurorehabilitation Hospitals. An MRI of the brain showed a significant lesion affecting the left arcuate fasciculus and, consequently, language and reading (Fig. 2).

She presented at that time with superficial acquired dyslexia with difficulties recognizing word forms. Her speech impairments included occasional anomia, semantic, phonemic and morphemic paraphasias, as well as paralexias and paragraphias in Portuguese. According to her family, she had even greater difficulties with Farsi. Before the stroke, TF had been fluent in the two languages and was able to speak, read, and write well in both. Her academic performance was good. She had returned to a Brazilian school three months after the stroke but was unable to return to an Iranian school. At that time, she presented with right hemiplegia, community gait and mixed muscle tonus with spasticity and involuntary movements.

TF's linguistic performance, before and after intervention, was assessed using the Montreal-Toulouse Language Assessment Battery for Aphasia - Brazilian Version (MTL-BR; Pagliarin et al., 2014) as well as the Brazilian Naming Test for Children, an adapted version of the Boston Naming Test (Miotto et al., 2010). General cognitive abilities were assessed using the Brazilian version of the Wechsler Intelligence Scale - IV (Wechsler, 2013). MRI data acquisition for the DTI was done with a 3T Siemens Magnetom Trio Tim MRI system (Siemens AG; Erlangen, Germany)

Neuropsychological rehabilitation lasted six months and included holistic therapy (Braga et al., 2012), as well as cognitive therapy that consisted of three 60-minute sessions per week to train executive functions such as working memory and cognitive flexibility. Speech therapy was also included and comprised two 60-minute sessions per week to target lexical access strategies and the use of lexical means for reading. The interventions were effective. In particular, behavioral performance in dictation, naming pictures, written naming, number dictation and written text comprehension increased significantly as revealed by the MTL-BR and the BNTC. Before the intervention, TF presented with a low average score on the WISC-IV Processing Speed test; after the intervention, she attained average performance on the same test. Social communication and reading improved significantly with normal or no-deficit 


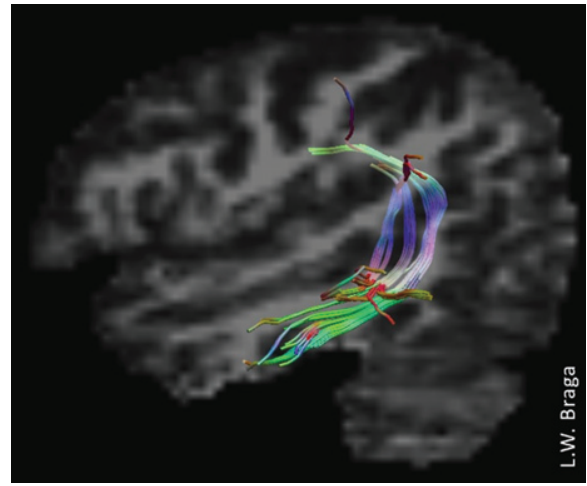

Fig 3a - Right Arcuate Fasciculus: Before Intervention

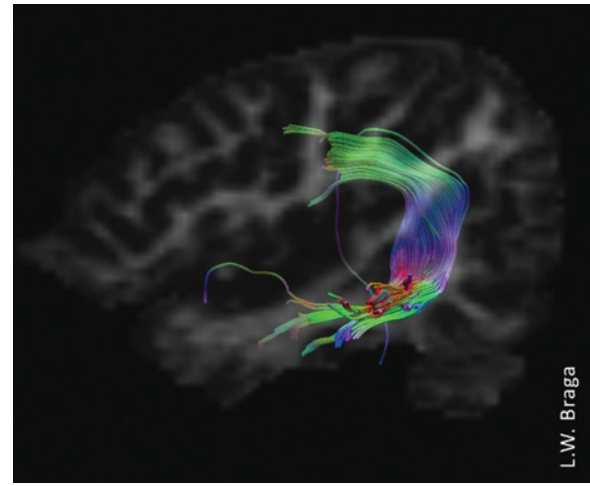

Fig 3b - Right Arcuate Fasciculus:

After intervention

Fig. 3. DTI images of TF's right cerebral hemisphere (a) before intervention and b) after intervention show a neurostructural increase in white matter. There was a significant increase in number of fibers, volume, and length of the right arcuate fasciculus after intervention.

scores and reading performance was attained in the final evaluation. The behavioral improvements were accompanied by significant neurostructural white matter changes in the arcuate fasciculus (AF). From the beginning to the end of therapy, the right-hemispheric AF showed increased FA values, number of fibers, volume and length, with mean diffusivity (MD) slightly decreasing accordingly (Fig. 3). Such a pattern was not observed for the left $\mathrm{AF}$, in which only slight changes of the values were observed.

These neuroimaging findings illustrate that a shortterm, but behaviorally effective training program can result in significant increase of white matter strength measures, which we used and which are reported to reflect better structural connectivity and myelination of existing fiber tracts (Brodt et al., 2018; Sagi et al., 2012). Specifically, the right-hemispheric AF has also been reported to reflect the success of rehabilitation processes in an aphasic single-case (Zipse et al., 2012). The authors reported that significantly improved behavioral performance in speech and language tasks was accompanied by an increased volume of the right AF. These studies provided evidence that neuropsychological rehabilitation activities can induce structural changes in the contralateral frontotemporal network homologous to the lesioned left-hemispheric language areas even in the chronic phase after stroke.

It is also important to note that increased righthemisphere processing following a left-hemispheric lesion has been interpreted by some investigators as maladaptive and a consequence of disinhibition (Naeser et al., 2005, Saur et al., 2006). However, in
TF's case the strength of the right AF increased in the context of continuous behavioral improvement, suggesting adequate right-hemispheric adaptation processes.

Providing evidence that neuropsychological rehabilitation after a brain injury results in measurable changes in brain function/connectivity allows for tangible, measurable evidence of meaningful biological change that help patients and their families adhere to a rehabilitation program protocol and is often required for securing financial backing for research. Present technology allows for visualizing the impact of interventions on the brain and can avoid the risk of creating a spiral of negative neuroplastic changes deriving from the disuse of previously healthy neural structures leading to accelerated aging, atrophy, and white matter alterations. Thus neuropsychological rehabilitation, coupled with neuroimaging evidence of neuroplasticity, suggests that one cannot only help remediate a disturbance in higher integrative brain function (Gaketti \& Saccio, 2017), but can avoid the development of maladaptive mechanisms involved in the recovery process.

\section{Diaschisis and neuroplasticity}

Influenced by the early work of von Monakow (1914), Luria (1948/1963) reasoned that cognitive impairments observed after traumatic brain injuries and cerebral vascular accidents were a function of at least two mechanisms. First, there was direct damage to brain tissue that rendered complex cognitive functions to be altered or impaired. Second, normal brain 
tissue not directly damaged may be rendered physiologically dysfunctional via the process of diaschisis. Carrera \& Tononi (2014) have reviewed historical and contemporary definitions of the term diaschisis and the research relating to it. A key concept of diaschisis is that a focal brain lesion can render adjacent as well as distal brain regions physiologically inactive. This is theoretically produced by some "interruption of the connections between the lesion and remote areas" (pg. 2409). When the interruption resolves, then normal neurophysiological activity resumes and improvement or "recovery" of a cognitive function is observed. Thus, the concept/phenomenon of diaschisis, by definition, is also a form of neuroplasticity and provides another model for understanding spontaneous recovery.

Luria et al. (1969) described several attempts to "de-inhibit" or restore "temporary inactivation of a functional system" (pg.370) in order to facilitate a return of "higher cortical functions." As noted earlier, this can be a very demanding task and often even intense behavioral efforts to do so produce minimal effects. Carrera \& Tononi's (2014) review of the literature suggests that perhaps different mechanisms underlie suppressed synaptic activity for cortico-cortico diaschisis versus corticosubcortico diaschisis. Recent research relating lesion location to functional outcome after stroke has highlighted the importance of cortical location and size of a lesion as well as the disturbance of white matter tracts. Neuroconnective disturbances were considered strong contributors to neuropsychological deficits (Siegel et al., 2016). The findings suggest that both cortico-cortico and cortico-subcortico synaptic disturbances are common even after relative focal brain lesions. Thus the complexity of diaschisis disturbances appears greater than originally considered. This may help explain the relative "resistance" of cortical mediated cognitive deficits to behavioral efforts at "de-inhibition." The fact that some form of neuropsychological recovery is often observed with time underscores the potential of neuroplasticity in neurorehabilitation for the adult brain, but it also reminds us of its present limitations.

External electrical stimulation of assumed normal surrounding neural tissue may permanently or temporally reduce the effects of diaschisis on various cognitive processes. In a recent meta-analysis and review of the effectiveness of language training for improving oral and written naming in patients with Primary Progressive Aphasia, Cotelli et al. (2020) noted, for example, that transcranial direct current stimulation administered just prior to language training tasks resulted in improved naming performance.

Sarasso et al. (2020) noted that EEG slowing is often observed in the "zone surrounding focal lesions" (pg.1) (e.g., an ischemic stroke). These investigators go on to demonstrate relatively different EEG slow wave activities in adjacent brain regions associated with focal ischemic injuries to the brain. Some of the EEG slowing patterns observed resembled slow wave sleep, while others were more compatible with the state of coma, and yet others represented the awake state. Their major theoretical point was that "local sleep-like reactivity" could be observed in the awake brain. However, there are also important implications for neuropsychological rehabilitation. First, the type of electrophysiological inhibition (i.e. diaschisis) associated with focal lesions may differ in the same person for different neuropsychological functions. Second, different "strengths" of diaschisis may be observed in different regions of the brain following different brain disorders. Third, the degree to which one can deinhibit neuronal activation patterns from a "sleep like pattern" to an awake pattern may correlate with the ability of the person to become progressively aware of a neuropsychological impairment that they only partially experience. This represents the classic disturbance of impaired self-awareness, which is a common barrier to successful neuropsychological rehabilitation (Prigatano \& Sherer, 2020).

\section{Can neuroimaging studies help individualize neuropsychological rehabilitation programs?}

While there is a growing consensus that neuroimaging studies before and after various forms of neurorehabilitation are important to advance our understanding of recovery or lack of recovery after a stroke (e.g. Liew et al., 2019), can these neuroimaging studies actually guide some aspects of neuropsychological rehabilitation? Braga (2017) noted that when training an illiterate adult to read, the student's progress was very slow and was associated with delayed construction of new neuronal networks in the reading area of the brain. Longitudinal neuroimaging revealed more robust progress in brain regions associated with phonetics, which led to changes in the teaching approach from syllabic to a phonetic method. This resulted in accelerated literacy acquisi- 
tion and was further associated with stronger neuronal networks in the corresponding cerebral areas.

Another intriguing case example has been reported by Errante et al. (2019). They described a prolonged course of rehabilitation for a young woman who suffered a severe traumatic brain injury (TBI). They noted that functional and structural MRI studies documented that there were preserved neural networks involved in motor and linguistic functioning despite substantial neuronal and white matter damage throughout the brain. The patient went on to show more motor and language functional recovery than was expected. They attribute that recovery to the intact neural networks. They suggested that detailed brain imaging may help clinicians determine what networks may be functional and thereby help the rehabilitationist direct rehabilitation activities toward probably intact networks. Cassidy \& Cramer (2017) make the same point when discussing the importance of "therapeutic-driven mechanisms of recovery." (pg. 33).

\section{Present realities and practical limitations}

As exciting as neuroimaging studies may be for neuropsychological and neurorehabilitation efforts, researchers and clinicians involved in both areas of study note the numerous limitations to our understanding of neuroplasticity and the interpretation of neuroimaging findings. The most commonly recognized problem is that most studies often involve few subjects as noted above. Subject populations are also notoriously heterogeneous. Intervention strategies vary greatly across rehabilitation centers and detailed descriptions of behavioral changes are often lacking. Furthermore, the magnitude of correlation between well-defined cognitive and behavioral changes with well-defined neuroimaging changes across studies is often lacking. It appears close to impossible to control for all of the variables that might influence the interpretation of findings.

As great as this challenge is, the ENIGMA Stroke Recovery Working Group (Liew et al., 2019) has gone to some lengths to describe a methodology for sharing neuroimaging and behavioral findings across national and international studies to provide "well-powered meta and mega-analytic approaches" to the study of recovery following stroke. A careful review of the methodological steps they are taking to harmonize neuroimaging and behavioral data across multiple national and international centers may help neuropsychologists plan similar research efforts in the future.

\section{Conclusion}

Traditionally holistic approaches to neuropsychological rehabilitation after brain injury have emphasized the importance of teaching patients to compensate for residual cognitive and motor difficulties. In this context, working with patients to emotionally adjust to permanent impairments was very important. Likewise, guiding them into a productive lifestyle with appropriate group support was crucial. This clinical commentary attempts to illustrate that we are now entering a new era in neuropsychological rehabilitation in which incorporating emerging scientific understanding of neuroplasticity, spontaneous recovery and diaschisis is needed to advance the field. In this regard, the active utilization of advanced neuroimaging technologies may not only help capture changes in brain structure, function and connections underlying recovery, but may (in some instances) help guide neuropsychological rehabilitation efforts. Case reports suggest this may be achieved by identifying neural networks that are only minimally effected by a given brain insult and developing neuropsychological interventions aimed at utilizing those intact networks to foster greater restoration of function. In this regard, techniques for reducing the complex effects of diaschisis may hold much promise as initially suggested by Luria (Luria, 1948/1963).

\section{Acknowledgments}

None.

\section{Conflict of interest}

None to report.

\section{References}

Abutalebi, J., Keim, R., Brambati, S. M., Tettamanti, M., Cappa, S. F., De Bleser, R., \& Perani, D. (2007). Late acquisition of literacy in a native language. Human Brain Mapping, 28(1), 19-33. https://doi.org/10.1002/hbm.20240.

Ardila, A., Bertolucci, P. H, Braga, L. W, Castro-Caldas, A., Judd, T., Kosmidis, M. H., Matute, E., Nitrini, R., Ostrosky-Sollis, F., \& Rosselli, M. (2010). Illiteracy: the neuropsychology of cog- 
nition without reading. Archives of Clinical Neuropsychology, 25(8), 689-712.

Ben-Yishay, Y., \& Diller, L. (2011). Handbook of Holistic Neuropsychological Rehabilitation. New York, NY: Oxford University Press.

Braga, L. W., Rossi, L., Moretto, A. L., da Silva, J. M., \& Cole, M. (2012). Empowering preadolescents with ABI through metacognition: preliminary results of a randomized clinical trial. NeuroRehabilitation, 30(3), 205-212.

Braga, L. W., Amemiya, E., Tauil, A., Suguieda, D., Lacerda, C., Klein, E., Dehaene-Lambertz, G., \& Dehaene, S. (2017). Tracking adult literacy acquisition with functional MRI: a single-case study. Mind Brain and Education, 11(3), 121-132.

Brodt, S., Gais, S., Beck, J., Erb, M., Scheffler, K., \& Schönauer, M. (2018). Fast track to the neocortex: A memory engram in the posterior parietal cortex. Science, 362(6418), 1045-1048.

Caeyenberghs, K., Clemente, A., Imms, F., Egan, G., Hocking, D. R., Leemans, A., Metzler-Baddeley, C., Jones, D. K., \& Wilson, P. H. (2018). Evidence for training-dependent structural neuroplasticity in brain-injured patients: A critical review. Neurorehabilitation and Neural Repair, 32(2), 99-114.

Carey, L. M, \& Seitz, R. J. (2007). Functional neuroimaging in stroke recovery and neurorehabilitation: conceptual issues and perspectives. International Journal of Stroke, 2(4), 245-264.

Carrera, E., \& Tononi, G. (2014). Diaschisis: Past, present, future. Brain, 137(9), 2408-2422.

Cassidy, J. M., \& Cramer, S. C. (2017). Spontaneous and therapeutic-induced mechanisms of functional recovery after stroke. Translational Stroke Research, 8(1), 33-46.

Castro-Caldas, A. R., \& Guerreiro, M. (1997). Neuropsychological aspects of illiteracy. Neuropsychological Rehabilitation, 7(4), 327-338.

Corbetta, M. (2008). Functional brain imaging and neurological recovery. In D. T. Stuss, G. Winocur, \& I. H. Robertson (Eds.), Cognitive Neurorehabilitation (Second ed., 162-181). Cambridge University Press.

Cotelli, M., Manenti, R., Ferrari, C., Gobbi, E., Macis, A., \& Cappa, S. F. (2020). Effectiveness of language training and non-invasive brain stimulation on oral and written naming performance in Primary Progressive Aphasia: A meta-analysis and systematic review. Neuroscience and Biobehavioral Reviews, 108, 498-525.

Cramer, S. C., Sur, M., Dobkin, B. H., O’Brien, C., Sanger, T. D., Trojanowski, J. Q., Rumsey, J. M., Hicks, R., Cameron, J., Chen, D., Chen, W. G., Cohen, L. G., deCharms, C., Duffy, C. J., Eden, G. F., Fetz, E. E., Filart, R., Freund, M., Grant, S. J., Haber, S., . . Vinogradov, S. (2011). Harnessing neuroplasticity for clinical applications. Brain: a Journal of Neurology, 134(Pt 6), 1591-1609.

Dehaene, S., Pegado, F., Braga, L. W., Ventura, P., Nunes Filho, G., Jobert, A., Dehaene-Lambertz, G., Kolinsky, R., Morais, J., \& Cohen, L. (2010). How learning to read changes the cortical networks for vision and language. Science, 330(6009), 13591364.

Dehaene, S., Pegado, F., Braga, L. W., Ventura, P., Nunes Filho, G., Jobert, A., Dehaene-Lambertz, G., Kolinsky, R., Morais, J., \& Cohen, L. (2011). L'imapct de l'apprentissage de la lecture sur le cerveau. Medecine Science (Paris), 27(3), 236-238.

Dehaene, S., Cohen, L., Morais, J., \& Kolinsky, R. (2015). Illiterate to literate: behavioural and cerebral changes induced by reading acquisition. Nature Reviews Neuroscience, 16(4), 234244.
De Schotten, M. T, Cohen, L., Amemya, E., Braga, L. W., \& Dehaene, S. (2014). Learning to read improves the structure of the arcuate fasciculus. Cerebral Cortex, 24(4), 989-995.

Errante, A., Saviola, D., Fasano, F., Basagni, B., Alinovi, S., Bosetti, S., Chiari, M., Minardi, R., Pinardi, C., Crisi, G., Fogassi, L., \& De Tanti, A. (2019). Application of an intensive rehabilitation program after very late recovery of consciousness: A single-case neurorehabilitation and neuroimaging study. Journal of Central Nervous System Disease, 11, 1179573519843492.

Gale, S. D., \& Pearson, C. M. (2012). Neuroimaging predictors of stroke outcome: implications for neurorehabilitation. Neurorehabilitation, 31(3), 331-344.

Galetto, V., \& Sacco, K. (2017). Neuroplastic changes induced by cognitive rehabilitation in traumatic brain injury: A review. Neurorehabilitation Neural Repair, 31(9), 800-813. https://doi:10.1177/1545968317723748

Goldstein, K. (1942). Aftereffects of brain injuries in war: Their evaluation and treatment: The application of psychologic methods in the clinic. New York, NY: Grune \& Stratton.

Goldstein, K. (1954). The concept of transference in treatment of organic and functional nervous disease. Acta psychotherapeutica, psychosomatica et orthopaedagogica, 2(3-4), 334-353.

Hamaide, J., Groof, G., \& Linden, A. V. (2016). Neuroplasticity and MRI: A perfect match. NeuroImage, 131, 13-28.

Heiss, W. D. (2017). Contribution of neuro-imaging for prediction of functional recovery after ischemic stroke. Cerebrovascular Disease, 44(5-6), 266-276.

Johansen-Berg, H. (2007). Functional imaging of stroke recovery: what have we learnt and where do we go from here? International Journal of Stroke, 2(1), 7-16.

Katz, D. I., \& Dwyer, B. (2021). Clinical neurorehabilitation: using principles of neurological diagnosis, prognosis, and neuroplasticity in assessment and treatment planning. Seminars in Neurology, 41(02), 111-123.

Liew, S. L., Zavaliangos-Petropulu, A., Jahanshad, N., Lang, C. E., Hayward, K. S., Lohse, K. R., Juliano, J. M., Assogna, F., Baugh, L. A., Bhattacharya, A. K., Bigjahan, B., Borich, M. R., Boyd, L. A., Brodtmann, A., Buetefisch, C. M., Byblow, W. D., Cassidy, J. M., Conforto, A. B., Craddock, R. C., Dimyan, M. A., ... Thompson, P. M. (2020). The ENIGMA Stroke Recovery Working Group: Big data neuroimaging to study brain-behavior relationships after stroke. Human brain mapping, 10.1002/hbm.25015.

Luria, A. R. (1948/1963). Restoration of function after brain trauma (in Russian). London: Academy of Medical Science/Pergamon.

Luria, A. R. Naydin, V.L., Tsvetkova, L. S., \& Vinarskaya, E. N. (1969). Restoration of higher cortical function following local brain damage. In P.J. Vinkin, \& G.W. Bruyn (Eds.), Handbook of clinical neurology (Vol. 3). Amsterdam; North-Holland.

Miotto, E. C., Sato, J., Lucia, M. C., Camargo, C. H., \& Scaff, M. (2010). Development of an adapted version of the Boston Naming Test for Portuguese speakers. Brazilian Journal of Psychiatry, 32(3), 279-282.

Muñoz-Cespedes, J. M., Rios-Lago, M., Paul, N., \& Maestu, F. (2005). Functional neuroimaging studies of cognitive recovery after acquired brain damage in adults. Neuropsychology Review, 15(4), 169-183.

Naeser, M. A., Martin, P. I., Nicholas, M., Baker, E. H., Seekins, H., Kobayashi, M., \& Pascual-Leone, A. (2005). Improved picture naming in chronic aphasia after TMS to part of right Broca's 
area: an open-protocol study. Brain and Language, 93(1), 95105.

Newcombe, F. (2002). An overview of neuropsychological rehabilitation: a forgotten past and a challenging future. In W. Brouwer, E. van Zomeren, I. Berg, A Bouma, E. de Haan, (Eds.). Cognitive Rehabilitation a Clinical Neuropsychological Approach, (23). Amsterdam, The Netherlands: Boom Publishers

Nordvik, J. E, Walle, K. M., Nyberga, C. K., Anders, M. F., Walhovdc K. B., Westlyeb, L. T, \& Tornas, S. (2014). Bridging the gap between clinical neuroscience and cognitive rehabilitation: the role of cognitive training, models of neuroplasticity and advanced neuroimaging in future brain injury rehabilitation. NeuroRehabilitation, 34(1), 81-85.

Pagliarin, K. C., Ortiz, K. Z., Parente, M. A. D. M. P., Arteche, A., Joanette, Y., Nespoulous, J. L., \& Fonseca, R. P. (2014). Montreal-Toulouse language assessment battery for aphasia: validity and reliability evidence. NeuroRehabilitation, 34(3), 463-471.

Prigatano, G. P. (1995). 1994 Sheldon Berrol, M.D., Senior Lectureship: The problem of lost normality after brain injury. Journal of Head Trauma Rehabilitation, 10(3), 87-95.

Prigatano, G. P. (2008). Neuropsychological rehabilitation and psychodynamic psychotherapy. In J. Morgan \& J. Ricker (Eds), Textbook of clinical neuropsychology, (985). New York, NY: Taylor \& Frances.

Prigatano, G. P., \& Sherer, M. (2020). Impaired self-awareness and denial during the postacute phases after moderate to severe traumatic brain injury. Frontiers in psychology, 11, 1569.

Ríos-Lago, M, Paúl-Lapedriza N., Muñoz-Céspedes, J. M., Maestú, F., Álvarez-Linera Prado, J., Ortiz, T. (2004). Aplicación de la neuroimagen funcional al estudio de la rehabilitación neuropsicológica. Revista de Neurologia, 38(4), 366-373.

Sagi, Y., Tavor, I., Hofstetter, S., Tzur-Moryosef, S., BlumenfeldKatzir, T., \& Assaf, Y. (2012). Learning in the fast lane: new insights into neuroplasticity. Neuron, 73(6), 1195-1203.

Sarasso, S., D'Ambrosio, S., Fecchio, M., Casarotto, S., Viganò, A., Landi, C., Mattavelli, G., Gosseries, O., Quarenghi, M., Laureys, S., Devalle, G., Rosanova, M., \& Massimini, M. (2020). Local sleep-like cortical reactivity in the awake brain after focal injury. Brain: a Journal of Neurology, 143(12), 3672-3684.
Saur, D., Lange, R., Baumgaertner, A., Schraknepper, V., Willmes, K., Rijntjes, M., \& Weiller, C. (2006). Dynamics of language reorganization after stroke. Brain, 129(Pt 6), 1371-1384.

Siegel, J. S., Ramsey, L. E., Snyder, A. Z., Metcalf, N. V., Chacko, R. V., Weinberger, K., Baldassarre, A., Hacker, C. D., Shulman, G. L., \& Corbetta, M. (2016). Disruptions of network connectivity predict impairment in multiple behavioral domains after stroke. Proceedings of the National Academy of Sciences of the United States of America, 113(30), E4367-E4376.

Stinear, C. M, \& Ward, N. S. (2013). How useful is imaging in predicting outcomes in stroke rehabilitation?. International Journal of Stroke, 8(1), 33-37.

Strangman, G., O’Neil-Pirozz, T. M, Burke, D., Cristina, D., Goldstein, R., Rauch, S. L., Savage, C. R., \& Glenn, M. (2005). Functional neuroimaging and cognitive rehabilitation for people with traumatic brain injury. American Journal of Physical Medicine and Rehabilitation, 84(1), 62-75.

Turkeltaub, P. E., Gareau, L., Flowers, D. L., Zeffiro, T. A., \& Eden, G. F. (2003). Development of neural mechanisms for reading. Nature Neuroscience, 6(7), 767-773.

von Monakow, C., Mott, F., \& Monakow, C. (1914). Die Lokalisation im Grosshirn und der Abbau der Funktion durch kortikale Herde. J.F. Bergmann.

Warren, D. E., Power, J. D., Bruss, J., Denburg, N. L., Waldron, E. J., Sun, H., Petersen, S. E., \& Tranel, D. (2014). Network measures predict neuropsychological outcome after brain injury. Proceedings of the National Academy of Sciences of the United States of America, 111(39), 14247-14252.

Wechsler, D. (2013). Escala Weschsler de inteligência para crianças: WISC-IV. Manual Técnico. (4. ed.). Casa do Psicólogo.

Zangwill, O. L. (1947). Psychological aspects of rehabilitation in cases of brain injury. British Journal of Psychology. General Section, 37(2), 60-69.

Zatorre, R. J., Fields, R. D., \& Johansen-Berg, H. (2012). Plasticity in gray and white: neuroimaging changes in brain structure during learning. Nature Neuroscience, 15(4), 528-536.

Zipse, L., Norton, A., Marchina, S., \& Schlaug, G. (2012). When right is all that is left: plasticity of right-hemisphere tracts in a young aphasic patient. Annals of the New York Academy of Sciences, 1252, 237-245. 